\title{
Influence of Temperature Variation on the Vibrational Characteristics of Fused Silica Cylindrical Resonators for Coriolis Vibratory Gyroscopes
}

\author{
Pengbo Xiao, Zhinan Qiu, Yiming Luo, Yao Pan*, Tianliang Qu, Kaiyong Yang, Hui Luo and \\ Shiqiao Qin * \\ College of Advanced Interdisciplinary Studies, National University of Defense Technology, \\ Changsha 410073, China; xiaopengbo09@nudt.edu.cn (P.X.); qiuzhinan17@nudt.edu.cn (Z.Q.); \\ luoyiming11@nudt.edu.cn (Y.L.); qutianliang@nudt.edu.cn (T.Q.); yky208@nudt.edu.cn (K.Y.); \\ luohui.luo@163.com (H.L.) \\ * Correspondence: panyao08@nudt.edu.cn (Y.P.); Sqqin8@nudt.edu.cn (S.Q.); \\ Tel.: +86-0731-8700-4196 (Y.P. \& S.Q.)
}

Received: 23 January 2020; Accepted: 12 February 2020; Published: 14 February 2020

\begin{abstract}
The fused silica cylindrical resonator is a type of axisymmetric resonator that can be used for Coriolis vibratory gyroscopes. Although the resonant frequency, frequency mismatch, and $\mathrm{Q}$ factor are natural properties of the resonator, they can change with temperature. Therefore, the temperature drift severely limits the detection accuracy and bias stability of the gyroscope. In this paper, the influence of temperature variation on the vibrational characteristics of fused silica cylindrical resonators was investigated. Experiments were performed on a fused silica cylindrical resonator coated with $\mathrm{Cr} / \mathrm{Au}$ films. It was shown that at the temperature range from $253.15 \mathrm{~K}$ to $353.15 \mathrm{~K}$, the resonant frequency linearly increased with temperature, the frequency mismatch remained unchanged, and the $\mathrm{Q}$ factor gradually increased till about $333.15 \mathrm{~K}$, when it began to decrease. Meanwhile, the change of thermoelastic damping with temperature may dominate the variation of $\mathrm{Q}$ factor at the temperature range from $253.15 \mathrm{~K}$ to $353.15 \mathrm{~K}$. This phenomenon was theoretically analyzed and the variation trends of results were consistent with the theoretical analysis. This study indicates that, for the fused silica cylindrical resonator, to discover the influence of temperature variation on the resonant frequency, frequency mismatch, and $\mathrm{Q}$ factor, there are certain rules to follow and repeat. The relationship between temperature and frequency can be established, which provides the feasibility of using self-calibration based on temperature characteristics of the resonator for temperature drift compensations. Additionally, there is an optimum temperature that may improve the performance of the Coriolis vibratory gyroscope with the fused silica cylindrical resonator.
\end{abstract}

Keywords: fused silica cylindrical resonator; frequency mismatch; Q factor; temperature; coriolis vibratory gyroscope

\section{Introduction}

Coriolis vibratory gyroscopes (CVGs), especially those with axisymmetric shell resonators, have many advantages including high reliability, considerable stability, long life, low power consumption, and a maintenance-free concept, and are widely used in navigation fields and platform stabilization systems [1-9]. For this type of gyroscope, there are mainly two types of axisymmetric shell resonators-hemispherical shell resonators and cylindrical shell resonators [10]. The representative products using hemispherical shell resonators include the Northrop Grumman H130 series and the Safran Regys series [11,12]. The Northrop Grumman Hubble hemispherical resonator gyroscope (HRG), in particular, has been reported to have bias stability of $0.00008^{\circ} / \mathrm{h}$ and angle random walk 
(ARW) of $0.00001^{\circ} / \mathrm{h}^{1 / 2}$ [8], and the Safran Regys 20 has been reported to have bias stability better than $0.01^{\circ} / \mathrm{h}$ [13]. The representative products using cylindrical shell resonators include the InnaLabs Inc. INL-CVG series; the INL-CVG-GU200, in particular, has been reported to have bias stability of $0.03^{\circ} / \mathrm{h}$ and ARW of $0.004^{\circ} / \mathrm{h}^{1 / 2}$ [14]. The University of Michigan has reported a micro birdbath shell resonator gyroscope with bias stability of $0.0103^{\circ} / \mathrm{h}$ [15]. The fused silica cylindrical shell resonators, in particular, are a type of high-performance resonators with high $Q$ factor, which have a simpler structure and are much easier to manufacture compared with hemispherical shell resonators. Therefore, they have broad applications in the tactical-level inertial navigation system [16]. Our research group has reported fused silica cylindrical resonators with the $Q$ factor approaching $10^{6}$ in 2016 [17] and $3 \times 10^{6}$ in 2019 [18].

For CVGs, the temperature effect is the main reason causing the variation of the output in addition to the input angular velocity $[19,20]$. The crucial vibrational characteristics of the resonator that limit the overall performance of the gyroscope, including the resonant frequency, frequency mismatch, and $Q$ factor, are all temperature-dependent [21]. Investigating the variation of vibrational characteristics with temperature is the first step for studying the temperature-drift compensations of CVGs. To give a few examples, $\mathrm{Li}$ et al. theoretically studied the output of hemispherical resonator gyroscope (HRG) under temperature influence, they obtained the frequency splitting formula and that the HRG has maximum disturbance limit in stable operation section [22]. Tang et al. discussed the static temperature characteristics of MEMS gyroscopes and derived that zero bias of the gyroscope is dependent on temperature and temperature gradient [23]. Therefore, gyros have better performance in environments with stable temperatures. Shao et al. experimentally investigated the resonant frequency and $Q$ factor of microscale hemispherical shell resonators under temperature influence. A linear and positive temperature coefficient of frequency was found, and the $Q$ factor showed an inverse trend at temperatures above $40^{\circ} \mathrm{C}$ [24]. Wu et al. investigated the temperature characteristics of vibration mode axes for metal cylindrical resonators. They found that a small frequency split and low thermal elastic modulus coefficient are necessary for the cylinder resonator [20]. Therefore, the influence of temperature variation on the resonant frequency and the $Q$ factor, there may be certain rules to follow. Wang et al. set up a mathematical model of the relationship between the temperature and the natural frequency of HRG, and compensations were made for the output drift of HRG based on natural frequency through a stepwise linear regression method [19], which shows the feasibility of making compensations based on the resonant frequency since the external temperature sensors may cause delays. Zega et al. investigated the frequency stability and thermoelastic effects for slotted tuning fork MEMS resonators (SETF). They theoretically computed the natural frequency and the quality factor of a MEMS resonator under different levels of material doping and different material orientations to obtain the analytical model of an idealized SETF. Meanwhile, they introduced slots in the resonator beams and applied an optimization tool to realize temperature stability together with a high quality factor [25]. Therefore, there are several means, like changing the levels of material doping, and changing the material orientation, which are helpful to mitigate the thermal drift of natural frequency of silicon MEMS resonators. Additionally, a careful design can help reduce the thermal drift even when slots are inserted in the devices in order to decrease thermoelastic losses. Our group has previously reported experimental results on the temperature-frequency characteristics of fused silica cylindrical resonators, and the linear relationship between temperature and resonant frequency was demonstrated [16], but the experimental results of other vibrational characteristics variations, for example, $Q$ factor and frequency mismatch variations, have not been reported. This paper intends to report the experimental results of the changes of resonant frequency, frequency mismatch, and $Q$ factor of fused silica cylindrical resonators with temperature, and provide theoretical analysis on these changes, showing the feasibility of using self-calibration based on temperature characteristics of the resonator to obtain the real-time temperature for compensations and providing an optimum temperature for performance improvement.

This paper comprises five sections. Using electrostatic excitation and detection, methods to measure the vibrational characteristics of fused silica cylindrical resonators with different temperature 
are described in Section 2. The influence of temperature variation on the vibrational characteristics of fused silica cylindrical resonators was theoretically analyzed in Section 3.1, while numerical calculations of resonant frequency, frequency mismatch, and damping loss with different temperatures are presented in Section 3.2. Experimental results and discussions are presented in Section 4. Section 5 concludes this paper with a summary of the results.

\section{Experiments and Methods}

In this research, a fused silica cylindrical resonator with a high $\mathrm{Q}$ factor was fabricated in the same way as stated in [17]. The sectional view of the resonator with electrodes is shown in Figure 1. The resonator has three main components, the resonant ring, the guide ring, and the bottom plate. The resonant ring has the maximum amplitude and is used for electrostatic excitation and detection, the guide ring is used for vibration transfer, and the bottom plate is used for support. The outer surface of the resonator was coated with $\mathrm{Cr} / \mathrm{Au}(\sim 20 / 60 \mathrm{~nm})$ film by magnetron sputtering and the resonator was rigidly connected with a fused silica base through its supporting rod. A fused silica cylindrical ring with laser-cut electrodes was attached on the base outside the resonator with a gap of approximately $16 \mu \mathrm{m}$. The main electrodes were used to excite or detect resonator vibration, while the auxiliary electrodes were grounded to reduce signal interference. The assembly was vacuum packaged with a pressure of $10^{-4} \mathrm{~Pa}$ with getter. During the experiment, the whole package was placed in a temperature-controlled chamber.

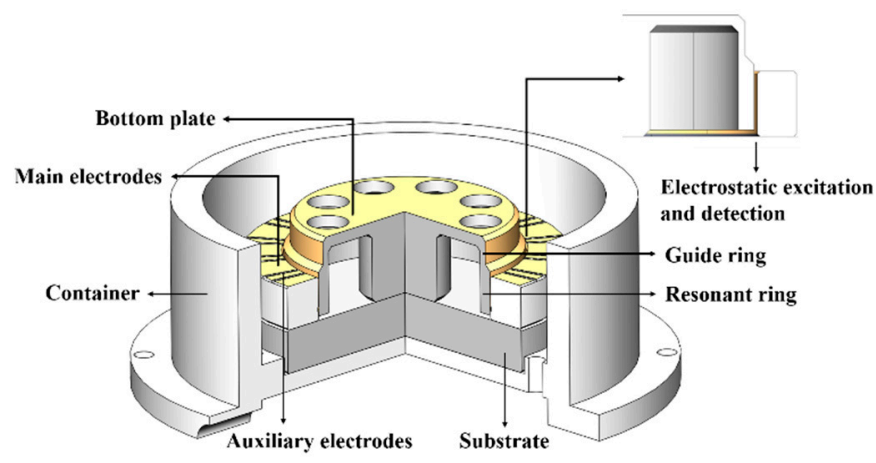

Figure 1. The sectional view of the resonator with electrodes.

\subsection{Electrostatic Excitation and Detection}

The outside metal film of the resonator and the main electrodes formed parallel plate capacitors used to excite or detect the vibration of the resonator from different directions. The constructions of electrostatic excitation and detection for fused silica resonators have been described in detail in $[26,27]$. Using a multifunctional I/O device, the actuating capacitors were connected to applied voltage sources. Excitation signals were generated by the multifunction I/O device with the controlled program designed and operated in the LabVIEW software, and all the relative parameters could be easily adjusted according to the actual conditions. Detection signals from sensing capacitors were collected by the multifunctional I/O device and processed by the LabVIEW program, as shown in Figure 2. 


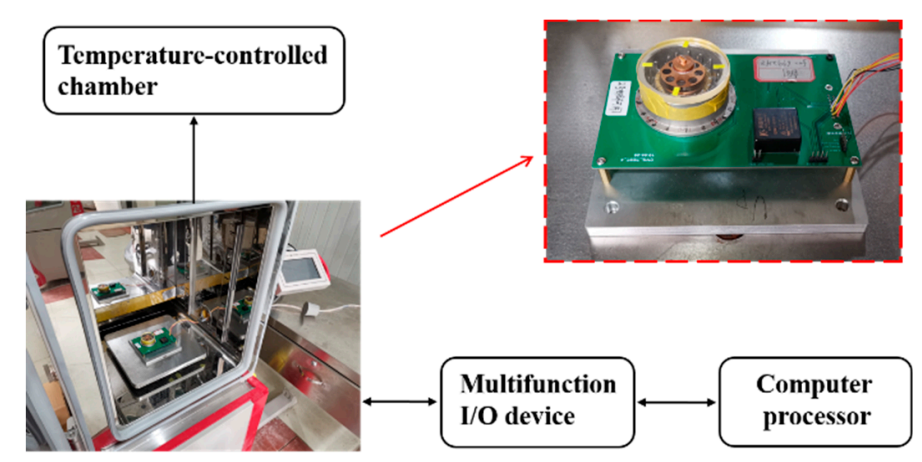

Figure 2. The diagram of the experimental setup used for the testing.

\subsection{Measurement of Vibrational Characteristics}

The temperature range was set from $253.15 \mathrm{~K}$ to $353.15 \mathrm{~K}$ with a $10 \mathrm{~K}$ step. Each time the temperature was adjusted, we waited about four hours for the resonator to reach a new equilibrium, then performed measurements of the vibrational characteristics. The cylindrical shell resonator works at $n=2$ resonant mode. Two principle axes have their own resonant frequencies $f 1$ and $f 2$, and they are equal, ideally. However, the shell thickness and surface state of the machine-made resonators are imperfect, which results in mass and stiffness distribution variations and causes differences between $f 1$ and $f 2$. Supposing $f 1>f 2$, therefore, the resonator has a pair of principal axes (the high-frequency principal axis, $f 1$ and the low-frequency principal axis, $f 2$ ), and the frequency split is defined as $f 1-f 2$, which is one of the main error sources in vibratory gyroscopes [28,29].

For the measurement of frequency and frequency mismatch, a pair of electrodes EA along with the low-frequency principal axis was used for actuation, while the pair ED in quadrature with EA was used for detection, as shown in Figure 3, and the structure shown has been modified for illustration purposes. A phase-locked loop (PLL) was applied to the detection signal. Meanwhile, an automatic gain control (AGC) loop was applied to realize the amplitude-control loop function, which maintained the amplitude constant. All control loops had already been programmed in the LabVIEW program previously mentioned. Therefore, the resonant frequency of the resonator was collected directly from the reference-phase loop. For the measurement of $Q$ factor, the ring-down method was employed. After the vibration was stabilized by the PLL and AGC loop, the actuation signal was cut off and the ring-down time constant $\tau$ was recorded. The relation between $Q$ factor and ring-down time constant is $Q=\pi f \tau$ [30], where $f$ is the resonant frequency of the resonator. The measurement for the resonant frequency was repeated for the high-frequency principal axis, hence the frequency mismatch was acquired.

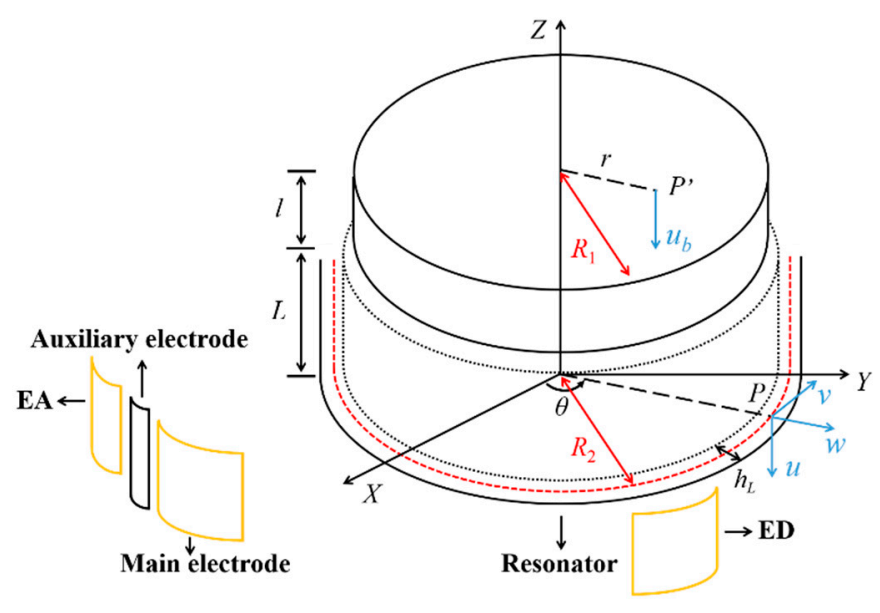

Figure 3. The structure diagram of the resonator with electrodes. 


\section{Theoretical Analysis}

The intrinsic mode in the form of a four-node standing wave is investigated in this paper, the vibration of a cylindrical shell resonator can be expressed according to [31,32]

$$
\begin{aligned}
& u=U(x) \cdot \cos (2 \theta) \cdot \cos (\omega t) \\
& v=V(x) \cdot \sin (2 \theta) \cdot \cos (\omega t) \\
& w=W(x) \cdot \cos (2 \theta) \cdot \cos (\omega t) \\
& u_{b}=U_{b}(r) \cdot \cos (2 \theta) \cdot \cos (\omega t)
\end{aligned}
$$

where $u, v$, and $w$ are respectively the tangential, axial, and radial displacement vector of a point $P$ on the shell's middle surface with the angular position $\theta$, and $u_{b}$ is the displacement vector of a point $P^{\prime}$ on the bottom plate with the radial position $r$, as shown in Figure 3. $U(x), V(x), W(x)$ and $U_{b}(r)$ are mode functions, which are presented in [33].

\subsection{Theoretical Analysis}

Since the stiffness of the resonator varies with the elastic modulus under varying temperatures, the resonant frequency of the resonator will also change. Assuming that the temperature-dependent elastic modulus of the resonator is [34-38]

$$
E(T)=E_{0}+k_{E T} E_{0}\left(T-T_{0}\right)
$$

where $E(T), E_{0}$ are respectively the elastic modulus of the resonator at temperature $T$ and $T_{0,} k_{E T}$ is the temperature coefficient for elastic modulus. Since the stiffness of the resonator is directly proportional to the elastic modulus, the stiffness variation induced by elastic modulus variation is

$$
\Delta K_{E}=k_{E T} K_{0}\left(T-T_{0}\right)
$$

where $K_{0}$ is the stiffness at temperature $T_{0}$. Considering the influence of thermal stress, the stiffness of the resonator becomes

$$
K=\left(K_{0}+\Delta K_{E}\right)(1+\alpha \Delta T)=K_{0}\left(1+\frac{E(T)-E_{0}}{E_{0}}\right)(1+\alpha \Delta T)
$$

where $\alpha$ is the thermal expansion coefficient and $\Delta T=T-T_{0}$. Therefore, the resonant frequency of the resonator at temperature $T$ is

$$
f_{T}=\sqrt{\frac{K}{m}}=\sqrt{\frac{K_{0}\left(1+\frac{E(T)-E_{0}}{E_{0}}\right)(1+\alpha \Delta T)}{m}}=f_{0} \sqrt{\frac{E(T)(1+\alpha \Delta T)}{E_{0}}}
$$

Substituting (2) into (5) and performing Taylor expansion on $x(\Delta T), f_{T}$ becomes

$$
\begin{aligned}
& f_{T}=f_{0} \sqrt{\frac{\left[E_{0}+k_{E T} E_{0}\left(T-T_{0}\right)\right](1+\alpha \Delta T)}{E_{0}}}=f_{0} \sqrt{\left[1+k_{E T} \Delta T\right](1+\alpha \Delta T)} \\
& =f_{0}[1+x(\Delta T)]^{1 / 2}=\left\{1+\frac{1}{2} x(\Delta T)-\frac{1}{8} x^{2}(\Delta T)+\mathrm{o}\left[x^{2}(\Delta T)\right]\right\}
\end{aligned}
$$

where $x(\Delta T)=\alpha k_{E T} \Delta T^{2}+\left(\alpha+k_{E T}\right) \Delta T$. Assuming the order of $\alpha$ and $k_{E T}$ are respectively about $10^{-7}$ and $10^{-4}$, and $\Delta T \leq 100 \mathrm{~K}, f_{T}$ approximates to

$$
\begin{aligned}
& f_{T}=f_{0}\left\{1+\frac{1}{2}\left[\alpha k_{E T} \Delta T^{2}+\left(\alpha+k_{E T}\right) \Delta T\right]-\frac{1}{8}\left[\alpha k_{E T} \Delta T^{2}+\left(\alpha+k_{E T}\right) \Delta T\right]^{2}\right\} \\
& =f_{0}\left[1+\frac{1}{2}\left(\alpha+k_{E T}\right) \Delta T\right]
\end{aligned}
$$


which indicates that the resonant frequency of the resonator linearly changes with temperature in this case. Meanwhile, assuming the resonant frequency excited in the low-frequency principal axis and the high-frequency principal axis at temperature $T_{0}$ are respectively $f_{01}$ and $f_{02}$ with an initial frequency mismatch of $\Delta f=f_{02}-f_{01}$, then the frequency mismatch $\Delta f_{T}=f_{T 02}-f_{T 01}$ at temperature $T$ equals to

$$
\Delta f_{T}=\left(f_{02}-f_{01}\right)\left[1+\frac{1}{2}\left(\alpha+k_{E T}\right) \Delta T\right]=f_{02}-f_{01}
$$

Therefore, the frequency mismatch of the resonator remains a constant in this case.

The total loss of a cylindrical shell resonator mainly consists of five types: air-damping correlated $1 / Q_{\text {gas }}$, support-loss correlated $1 / Q_{\text {sup }}$, surface-loss correlated $1 / Q_{\text {sur }}$, thermoelastic-damping correlated $1 / Q_{t h r}$, and internal-friction correlated $1 / Q_{\text {fri }}$, as described by the following Equation (10),

$$
\frac{1}{Q}=\frac{1}{Q_{\text {air }}}+\frac{1}{Q_{\text {sup }}}+\frac{1}{Q_{\text {sur }}}+\frac{1}{Q_{\text {thr }}}+\frac{1}{Q_{\text {fri }}}
$$

Since the fused silica cylindrical resonator was sealed in a vacuum packing container with a pressure of $10^{-4} \mathrm{~Pa}$ and the resonator was rigidly connected with the fused silica base, we neglected the air damping and the support loss in this research.

The resonator has a damaged layer on its surface due to machining and polishing. Such a damaged layer consists of the residual carbon of diamond paste and a hundred micron-thick layer of dislocations. It has inhomogeneous heat during vibration and leads to surface loss. According to the surface loss of the cylinder described in [39], the surface defect loss correlated $1 / Q_{\text {sur }}$ of the cylindrical resonator approximates to

$$
\frac{1}{Q_{\text {sur }}}=2 h_{\text {dam }} \cdot\left(\frac{1}{L}+\frac{1}{l}+\frac{1}{R_{1}}+\frac{1}{R_{2}}\right) \cdot \frac{E T \alpha^{2}}{c} \cdot \frac{2 \pi f_{T} t}{1+\left(2 \pi f_{T} t\right)^{2}}
$$

where $L, l$ are respectively the height of the resonant ring and the guide ring of the resonator, $R_{1}$ and $R_{2}$ are respectively the radiuses of middle surfaces of the guide ring and the resonant ring, $h_{d a m}$ is the depth of the damaged layer, $E$ is the elastic modulus, and $\alpha$ is the thermal expansion coefficient. The characteristic time $t$ is introduced by Zener [40] as "relaxation time" to describe anelasticity in solids, and is derived as $t=\psi^{2} \frac{c}{\kappa}$, where $\psi$ is the typical size of heat distribution [39], $\kappa$ is the thermal conductivity coefficient, and $c$ is the heat capacity.

The thermoelastic damping is a type of internal friction and the dissipation mechanism results from thermal conduction due to the temperature gradient in the fused silica. According to the thermoelastic damping of the cylindrical shell given by [40-42], the thermoelastic damping correlated $1 / Q_{t h r}$ of the resonant ring is

$$
\frac{1}{Q_{t h r 1}}=2\left|\frac{\operatorname{Im}\left(\omega_{m n}\right)}{\operatorname{Re}\left(\omega_{m n}\right)}\right|=2\left|\tan \frac{\Xi}{2}\right|
$$

where $\operatorname{Re}\left(\omega_{m n}\right)=\frac{1}{R_{2}} \sqrt{\frac{E}{\rho}} \sqrt{\psi} \cos \frac{\Xi}{2}, \operatorname{Im}\left(\omega_{m n}\right)=\frac{1}{R_{2}} \sqrt{\frac{E}{\rho}} \sqrt{\psi} \sin \frac{\Xi}{2}, \rho$ is the density, and $\Xi=$ $\arctan \left(\frac{\operatorname{Im}\left(\Delta \Omega^{2} m n\right)}{\operatorname{Re}\left(\Delta \Omega^{2} m n\right)+\Omega^{2} m n}\right)$. The parameters $\operatorname{Im}\left(\Delta \Omega^{2}{ }_{m n}\right), \operatorname{Re}\left(\Delta \Omega^{2}{ }_{m n}\right)$, and $\Omega^{2}{ }_{m n}$ are calculated in [41]. Similarly, the thermoelastic damping correlated $1 / Q_{t h r 1}^{\prime}$ of the guide ring is obtained, and the thermoelastic-damping correlated $1 / Q_{t h r 2}$ of the bottom plate is given by [43],

$$
\frac{1}{Q_{t h r 2}}=D\left[\frac{6}{\delta^{2}}-\frac{6}{\delta^{3}}\left(\frac{\sinh \delta+\sin \delta}{\cosh \delta+\cos \delta}\right)\right]
$$

where $D=\frac{(1+\mu) E \alpha^{2} T}{(1-2 \mu) \rho c}, \delta=\frac{h_{b}^{3 / 2}}{2 R_{1}}\left(\frac{10.21 c}{\kappa}\right)^{1 / 2}\left[\frac{E \rho}{3\left(1-\mu^{2}\right)}\right]^{1 / 4}, \mu$ is the Poisson's ratio, and $h_{b}$ is the thickness of the bottom plate. Therefore, the thermoelastic-damping correlated $1 / Q_{t h r}$ of the resonator equals to $1 / Q_{t h r 1}+1 / Q_{t h r 1}^{\prime}+1 / Q_{t h r 2}$. 
The internal friction is an energy loss in virtue of imperfect elastic stress, which is rendered irrecoverable, or unavailable for the resonator vibration. The internal-friction correlated $1 / Q_{\text {fri }}$ of the resonator is

$$
\frac{1}{Q_{f r i}}=\frac{U_{f r i_{1}}+U_{f r i_{2}}+U_{f r i_{3}}}{2 \pi S}
$$

where $S$ is the potential energy of the resonator and the explicit expression of $S$ is described in $[33,44]$, $U_{f r i_{1}}, U_{f r i_{2}}$, and $U_{f r i_{3}}$ are respectively the internal-friction loss of the radial, axial, and bottom plate of the resonator, and their explicit expressions are given according to the internal friction of cupped resonator described in [33]

$$
\begin{aligned}
& U_{f r i_{1}}=\frac{1}{24} \frac{E \xi h_{L}^{3}}{R_{2}^{3}} \int_{0}^{L} \int_{0}^{2 \pi}\left(\frac{\partial^{2} v}{\partial \theta \partial t}-\frac{\partial^{3} w}{\partial^{2} \theta \partial t}\right) \cdot\left(\frac{\partial v}{\partial \theta}-\frac{\partial^{2} w}{\partial^{2} \theta}\right) d \theta d x \\
& +\frac{1}{24} \frac{E \xi h_{l}^{3}}{R_{1}^{3}} \int_{L}^{L+l} \int_{0}^{2 \pi}\left(\frac{\partial^{2} v}{\partial \theta \partial t}-\frac{\partial^{3} w}{\partial^{2} \theta \partial t}\right) \cdot\left(\frac{\partial v}{\partial \theta}-\frac{\partial^{2} w}{\partial^{2} \theta}\right) d \theta d x \\
& U_{f r i_{2}}=\frac{1}{24} E R_{2} \xi h_{L}^{3} \int_{0}^{L} \int_{0}^{2 \pi} k_{x} \cdot \frac{d k_{x}}{d t} d \theta d x+\frac{1}{24} E R_{1} \xi h_{l}^{3} \int_{L}^{L+l} \int_{0}^{2 \pi} k_{x} \cdot \frac{d k_{x}}{d t} d \theta d x \\
& U_{f r i_{3}}=\frac{1}{24} E \xi h_{b} \int_{r_{0}}^{R_{1}} \int_{0}^{2 \pi} k_{y} \cdot \frac{d k_{y}}{d t} \cdot r d \theta d r
\end{aligned}
$$

where $\xi$ is the internal friction coefficient of the material, $h_{L}$ and $h_{l}$ are respectively the thickness of the resonant ring and the guide ring, $k_{x}=\frac{W(\mathrm{x})^{\prime \prime} \cos 2 \theta \cos (\omega t)}{\left\{1+\left[W(\mathrm{x})^{\prime} \cos 2 \theta\right]^{2}\right\}^{3 / 2}}$, and $k_{y}=\frac{U_{b}(r)^{\prime \prime} \cos 2 \theta \cos (\omega t)}{\left\{1+\left[U_{b}(r)^{\prime} \cos 2 \theta\right]^{2}\right\}^{3 / 2}}$.

\subsection{Numerical Calculations}

Table 1 presents some mechanical and thermal properties of fused silica used for the following numerical calculations, and the effects of temperature on elastic modulus, $E$, and Poisson's ratio, $\mu$, of fused silica are depicted in [36,37]. At the temperature range from $253.15 \mathrm{~K}$ to $353.15 \mathrm{~K}$, the elastic modulus and the Poisson's ratio both vary linearly with temperature as in $E(T)=1.267 \times 10^{7} \mathrm{~T}+$ $6.995 \times 10^{10}$ and $\mu(T)=1 \times 10^{-4} T+0.14[36,37]$, and other properties were considered as constants in this study. Table 2 presents the geometry parameters of the resonator. With these parameters, we can calculate the changes of specific vibrational characteristics with temperature according to the theoretical analysis in Section 3.1. According to Equation (5), numerical results show that the fitted value of resonant frequency is $f=0.6021 T+6812.2$, and the goodness of fit of the fitting curve is 1 , as shown in Figure 4. Meanwhile, according to Equation (7), the approximation of resonant frequency is $f=0.6036 T+6811.8$ and the relative difference of the gradient is $0.25 \%$, which indicates the reliability of the approximation.

Table 1. Mechanical and thermal properties of fused silica.

\begin{tabular}{ccc}
\hline Component & Value & Units \\
\hline Density, $\rho$ & $2.203 \times 10^{3}$ & $\mathrm{Kg} / \mathrm{m}^{3}$ \\
Damaged layer depth, $h_{\text {dam }}$ & 100 & $\mu \mathrm{m}$ \\
Heat capacity, $c$ & 772 & $\mathrm{JKg}^{-1} \mathrm{~K}^{-1}$ \\
Thermal conductivity, $\kappa$ & 1.39 & $\mathrm{~W} / \mathrm{mK}^{-1}$ \\
Thermal expansion coefficient, $\alpha$ & $5 \times 10^{-7}$ & $\mathrm{~K}^{-1}$ \\
Typical size of heat distribution, $\psi$ & $1 \times 10^{-6}$ & $\mathrm{~m}$ \\
Internal friction coefficient, $\xi$ & $1 \times 10^{-11}$ & \\
\hline
\end{tabular}


Table 2. Geometry parameters of the cylindrical resonator.

\begin{tabular}{ccc}
\hline Component & Value & Units \\
\hline Radius of the guide ring, $R_{1}$ & 12.3 & $\mathrm{~mm}$ \\
Radius of the resonant ring, $R_{2}$ & 12.6 & $\mathrm{~mm}$ \\
Height of the resonant ring, $L$ & 5.7 & $\mathrm{~mm}$ \\
Height of the guide ring, $l$ & 3.1 & $\mathrm{~mm}$ \\
Thickness of the resonant ring, $h_{L}$ & 1.2 & $\mathrm{~mm}$ \\
Thickness of the guide ring, $h_{l}$ & 0.5 & $\mathrm{~mm}$ \\
Thickness of the bottom plate, $h_{b}$ & 0.8 & $\mathrm{~mm}$ \\
\hline
\end{tabular}

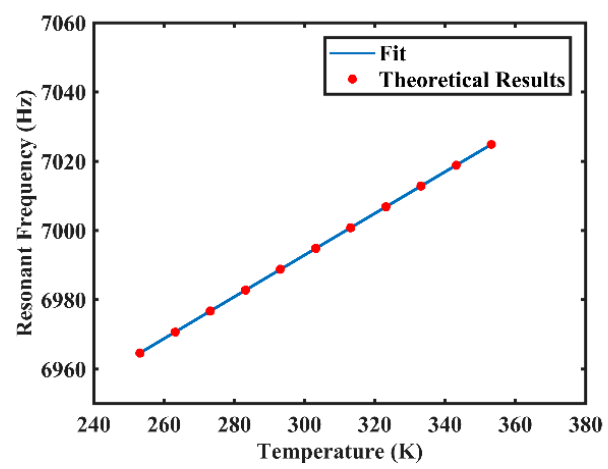

Figure 4. The fitted value of resonant frequency varying with temperature.

Different types of damping, varying with temperature at the temperature range from $253.15 \mathrm{~K}$ to $353.15 \mathrm{~K}$, were calculated and results are shown in Figure 5. According to Equation (10), the numerical calculation of surface-loss correlated $1 / Q_{\text {sur }}$ varying with temperature is shown in Figure $5 \mathrm{a}$. The surface loss increases linearly from about $0.9 \times 10^{-9}$ to $1.3 \times 10^{-9}$ (increased by $44.44 \%$ ) at the temperature range from $253.15 \mathrm{~K}$ to $353.15 \mathrm{~K}$. According to Equations (11) and (12), the numerical calculation of thermoelastic-damping correlated $1 / Q_{t h r}$ varying with temperature is shown in Figure $5 \mathrm{~b}$. The thermoelastic damping gradually decreases from about $2.38 \times 10^{-8}$ to $1.72 \times 10^{-8}$ (decreased by $27.73 \%$ ) at the temperature range from $253.15 \mathrm{~K}$ to $353.15 \mathrm{~K}$. According to Equations (13) and (14), the numerical calculation of internal-friction correlated $1 / Q_{\text {fri }}$ varying with temperature is shown in Figure 5c. The internal friction increases linearly from about $2.96 \times 10^{-7}$ to $3.01 \times 10^{-7}$ (increased by $1.66 \%$ ) at the temperature range from $253.15 \mathrm{~K}$ to $353.15 \mathrm{~K}$. Putting these three types of damping loss together, the numerical calculation of $1 / Q_{\text {sur }}+1 / Q_{t h r}+1 / Q_{\text {fri }}$ varying with temperature is shown in Figure $5 \mathrm{~d}$. The total loss gradually decreases from about $3.204 \times 10^{-7}$ to $3.192 \times 10^{-7}$ till about $328 \mathrm{~K}$ when it begins to rise and reaches $3.193 \times 10^{-7}$ at $353.15 \mathrm{~K}$. 


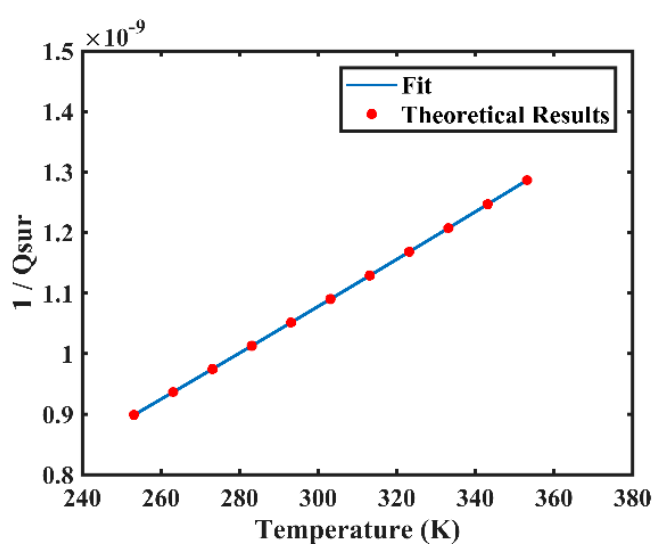

(a)

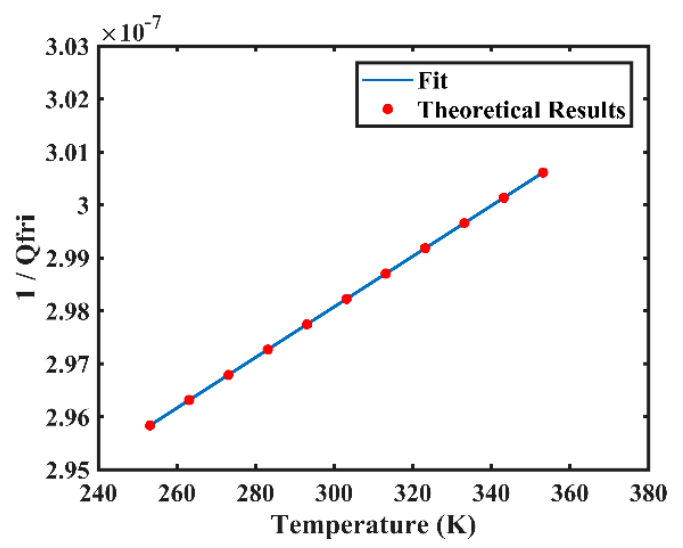

(c)

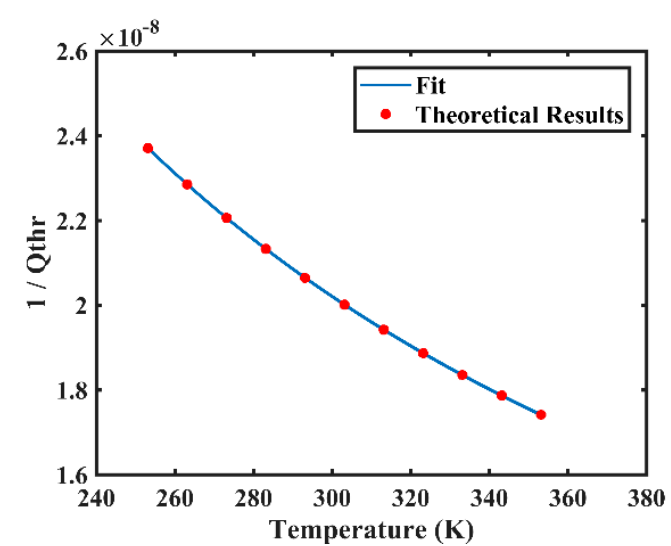

(b)

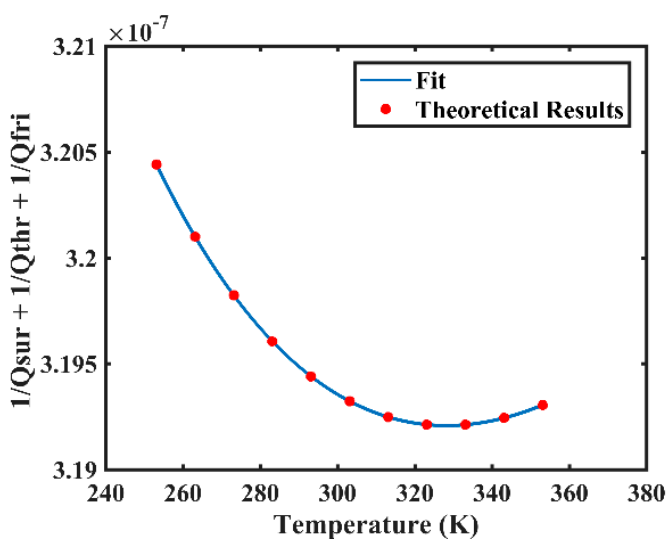

(d)

Figure 5. Numerical calculations of different types of damping loss varying with temperature at the temperature range from $253.15 \mathrm{~K}$ to $353.15 \mathrm{~K}$ : (a) the surface-loss correlated $1 / Q_{\text {sur }}$ varies with temperature; (b) the thermoelastic-damping correlated $1 / Q_{t h r}$ varies with temperature; (c) the internal-friction correlated $1 / Q_{f r i}$ varies with temperature; (d) the sum of $1 / Q_{\text {sur }}, 1 / Q_{t h r}$, and $1 / Q_{f r i}$ varies with temperature.

Therefore, at the temperature range from $253.15 \mathrm{~K}$ to $353.15 \mathrm{~K}$, the resonant frequency increases linearly, the frequency mismatch remains unchanged, and the total damping loss gradually decreases till about $328 \mathrm{~K}$ when it begins to rise.

\section{Experimental Results and Discussion}

The resonant frequency of the resonator excited in the low-frequency principal axis was measured during both the heating process and the cooling process-results are shown in Figure 6. Figure 6a shows that the fitted value of experimental resonant frequency in the heating process is $f_{1}=0.6196 \mathrm{~T}+6807.9$, and the goodness of fit of the fitting curve is 0.9998 . Figure $6 \mathrm{~b}$ shows that the fitted value of experimental resonant frequency in the cooling process is $f_{1}=0.6193 T+6808.1$, and the goodness of fit of the fitting curve is 0.9997 . The error of experimental results between two processes may result from the accuracy level of the temperature-controlled chamber, about $\pm 0.1 \mathrm{~K}$, which may cause a frequency fluctuation of $0.12 \mathrm{~Hz}$. Compared with the theoretical result $f=0.6021 T+6812.2$, the relative differences of the gradient in the heating process and the cooling process are respectively $2.82 \%$ and $2.78 \%$, which shows good consistency and repeatability. The error between experimental and theoretical results may result from the accuracy of mechanical and thermal properties used for theoretical calculations, for example, the elastic modulus, $E$ has been approximated to linear change at the temperature range from $253.15 \mathrm{~K}$ to $353.15 \mathrm{~K}$. Therefore, the resonant frequency is positively correlated with temperature 
and changes linearly at the temperature range from $253.15 \mathrm{~K}$ to $353.15 \mathrm{~K}$. Considering that each temperature only corresponds to one resonant frequency, the resonant frequency of the resonator can be used as a real-time measure of temperature variation to compensate for the performance of CVG with the temperature-dependent gyro drift and the temperature-dependent noise. In addition, the experimental results of the frequency mismatch at different temperatures remain at $0.056 \mathrm{~Hz}$, which are also consistent with theoretical analysis.

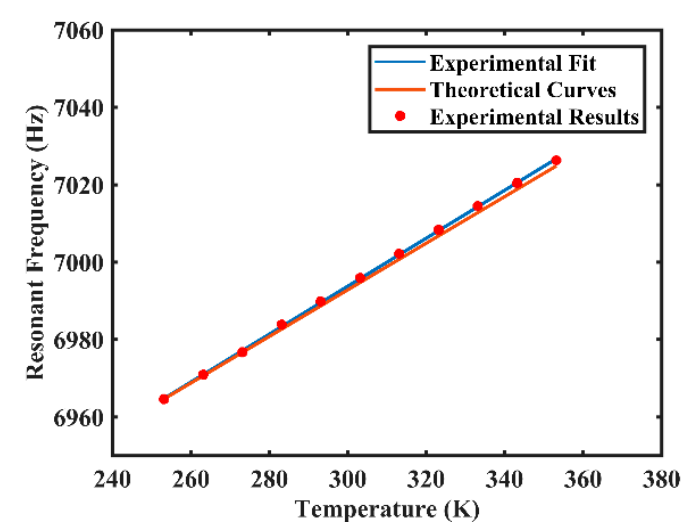

(a)

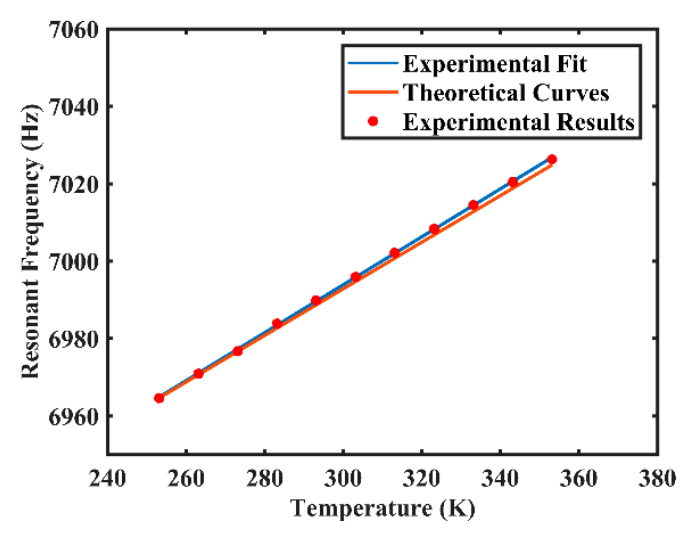

(b)

Figure 6. The experimental results of resonant frequency varying with temperature at the temperature range from $253.15 \mathrm{~K}$ to $353.15 \mathrm{~K}$ : (a) results from the heating process; (b) results from the cooling process.

The ring-down time constant measurement of the resonator in $253.15 \mathrm{~K}$ is shown in Figure 7, and the result is normalized with respect to the ring-down time constant at $333.15 \mathrm{~K}$. It is shown that the normalized ring-down time constant (low-frequency axis excited) $\tau_{\text {nor }}$ of the resonator at $253.15 \mathrm{~K}$ is about 0.801 .

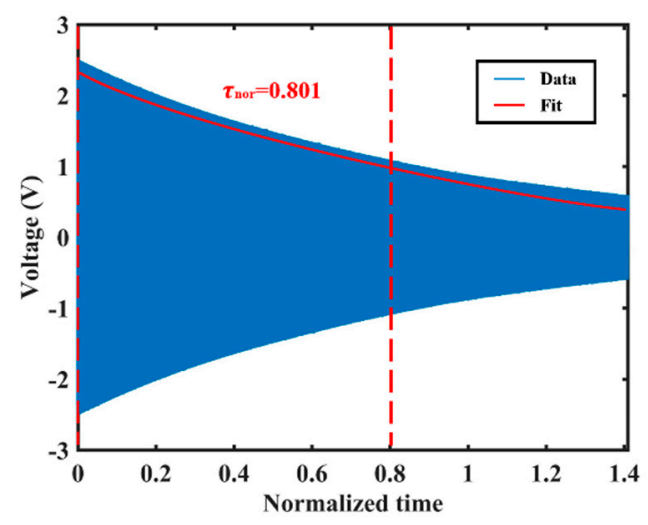

Figure 7. The measured normalized ring-down time constant of the resonator in $253.15 \mathrm{~K}$.

The measurement for the normalized ring-down time constant was repeated for different temperatures, hence the $\mathrm{Q}$ factor of the resonator varying with temperature is acquired and the results are normalized with respect to the $Q$ factor at $333.15 \mathrm{~K}$, as shown in Figure 8. The normalized $\mathrm{Q}$ factor of the resonator in the heating process and the cooling process both show an upward trend from $253.15 \mathrm{~K}$ to about $333.15 \mathrm{~K}$ when it reaches the top, and it gradually decreases till $353.15 \mathrm{~K}$, which shows good repeatability. The inverse of theoretical $1 / Q_{s u r}+1 / Q_{t h r}+1 / Q_{f r i}$ in Figure $5 \mathrm{~d}$ is also normalized with respect to the data at $333.15 \mathrm{~K}$ and displayed in Figure 8, which shows nearly the same trend and variation rate with the experimental curves. The error may result from the air damping, support loss, and other losses that have been neglected-although they are tiny and hardly vary with temperature, these losses still influence the exact value of $Q$ factor. Therefore, there should 
be an optimal temperature for minimum damping loss, and the resonator may reach the highest $Q$ factor at a certain temperature, which are consistent with the theoretical calculations. Meanwhile, according to the variation rate of different types of damping loss between two temperatures, the change of thermoelastic damping with temperature may dominate the variation of $Q$ factor at the temperature range from $253.15 \mathrm{~K}$ to $353.15 \mathrm{~K}$.

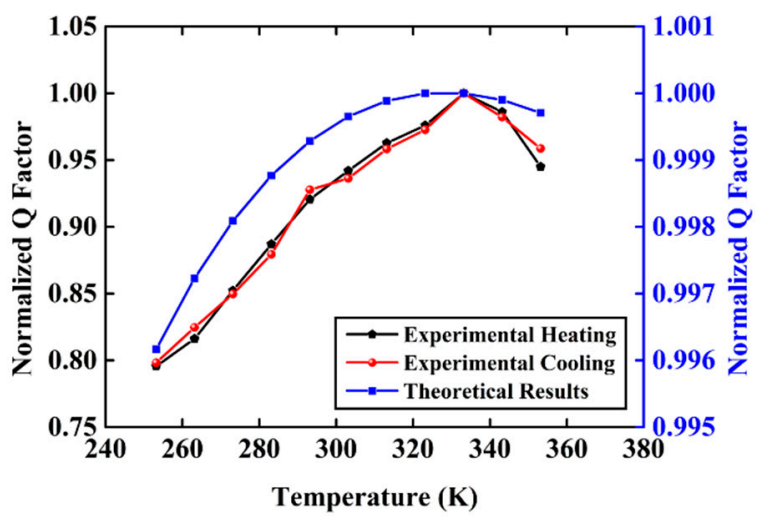

Figure 8. The measured and theoretical normalized $Q$ factor varying with temperature at the temperature range from $253.15 \mathrm{~K}$ to $353.15 \mathrm{~K}$.

\section{Conclusions}

This paper reports the experimental results of the changes in resonant frequency, frequency mismatch, and $Q$ factor under temperature influence. Experiments were performed on a film-coated fused quartz cylindrical resonator with ring electrodes. The resonant frequency increases linearly, while the frequency mismatch remains unchanged with temperature at the temperature range from $253.15 \mathrm{~K}$ to $353.15 \mathrm{~K}$. Meanwhile, the Q factor of the resonator gradually increases from $253.15 \mathrm{~K}$ till $333.15 \mathrm{~K}$, when it shows a downward trend, and the $\mathrm{Q}$ factor may reach its maximum at a temperature between $323.15 \mathrm{~K}$ and $343.15 \mathrm{~K}$. Meanwhile, the change of thermoelastic damping with temperature may dominate the variation of $Q$ factor at the temperature range from $253.15 \mathrm{~K}$ to $353.15 \mathrm{~K}$. All the vibrational characteristics were measured both in the heating process and the cooling process, and the results showed good repeatability. These changes were theoretically analyzed by introducing the effects of temperature on some mechanical and thermal properties of fused silica, for example, elastic modulus and Poisson's ratio into the resonant frequency and damping loss equations of the cylindrical resonator, and variation trends in experimental results were consistent with the theoretical analysis. Therefore, the relationship between temperature and resonant frequency is established, the resonant frequency of the resonator can be used as a real-time measure of temperature variation to compensate for the performance of a CVG with the temperature-dependent gyro drift and the temperature-dependent noise. In addition, there is an optimum temperature that may improve the performance of a CVG with the fused silica cylindrical resonator.

Author Contributions: Conceptualization, P.X., Y.P. and T.Q. Data curation, P.X., Z.Q. and Y.L. Formal analysis, K.Y. and H.L. Funding acquisition, T.Q., K.Y. and H.L. Investigation, P.X., Y.P. and S.Q. Methodology, P.X., Y.P. and S.Q. Project administration, K.Y. and H.L. Resources, P.X. and Z.Q. Software, P.X. and Z.Q. Validation, P.X., Y.P. and T.Q. Visualization, P.X. and Y.P. Writing-review \& editing, Y.P., T.Q. and S.Q. All authors have read and agreed to the published version of the manuscript.

Funding: This research was funded by the National Natural Science Foundation of China (Grant No. 11304384, 61575220).

Acknowledgments: The authors would like to thank the people who helped in mechanical processing.

Conflicts of Interest: The authors declare no conflicts of interest. 


\section{References}

1. Chikovani, V.V.; Yatzenko, Y.A.; Kovalenko, V.A. Coriolis Force Gyroscope with High Sensitivity. U.S. Patent 7513156B2, 7 April 2009.

2. Chikovani, V.; Yatsenko, Y.A.; Barabashov, A.; Marusyk, P.; Umakhanov, E.; Taturin, V.N. Improved accuracy metallic resonator CVG. IEEE Aerosp. Electron. Syst. Mag. 2009, 24, 40-43. [CrossRef]

3. Chikovani, V.; Okon, I.; Barabashov, A.; Tewksbury, P. A set of high accuracy low cost metallic resonator CVG. In Proceedings of the 2008 IEEE/ION Position, Location and Navigation Symposium, Monterey, CA, USA, 5-8 May 2008; pp. 238-243.

4. Sarapuloff, S. Dynamics of Precise Solid-State Gyroscopes of HRG and CRG Types. In Proceedings of the V-th Brazilian Symposium on Inertial Engineering (V SBEIN), Rio de Janeiro, Brazil, 27-29 November 2007; pp. 27-29.

5. Sarapuloff, S. Development and cost reduction of high-Q dielectric resonators of solid-state gyroscopes. In Proceedings of the 8 th Saint Petersburg International Conference on Integrated Navigation Systems, St. Petersburg, Russia, 28-30 May 2001; pp. 124-126.

6. Yatsenko, Y.A.; Petrenko, S.; Vovk, V.; Chikovani, V. Technological aspects of manufacturing of compound hemispherical resonators for small-sized vibratory gyroscopes. In Proceedings of the 6 th Saint Petersburg International Conference on Integrated Navigation Systems, St. Petersburg, Russia, 24-26 May 1999.

7. Watson, W.S. Vibratory gyro skewed pick-off and driver geometry. In Proceedings of the IEEE/ION Position, Location and Navigation Symposium, Indian Wells, CA, USA, 4-6 May 2010; pp. 171-179.

8. Rozelle, D.M. The hemispherical resonator gyro: From wineglass to the planets. In Proceedings of the 19th AAS/AIAA Space Flight Mechanics Meeting, Savannah, GA, USA, 8-12 February 2009; pp. 1157-1178.

9. Luo, Y.; Qu, T.; Zhang, B.; Pan, Y.; Xiao, P. Simulations and Experiments on the Vibrational Characteristics of Cylindrical Shell Resonator Actuated by Piezoelectric Electrodes with Different Thicknesses. Shock Vib. 2017, 2017, 2314858. [CrossRef]

10. Pan, Y.; Qu, T.; Wang, D.; Wu, S.; Liu, J.; Tan, Z.; Yang, K.; Luo, H. Observation and analysis of the quality factor variation behavior in a monolithic fused silica cylindrical resonator. Sens. Actuators A Phys. 2017, 260, 81-89. [CrossRef]

11. Jeanroy, A.; Bouvet, A.; Remillieux, G. HRG and marine applications. Gyroscopy Navig. 2014, 5, 67-74. [CrossRef]

12. Jeanroy, A.; Grosset, G.; Goudon, J.-C.; Delhaye, F. HRG by Sagem from laboratory to mass production. In Proceedings of the 2016 IEEE International Symposium on Inertial Sensors and Systems, Laguna Beach, CA, USA, 22-25 February 2016; pp. 1-4.

13. Safran. Available online: https://www.safran-group.com/ (accessed on 13 February 2020).

14. Beitia, J.; Fell, C.; O’Leary, J.; Okon, I.; Simonenko, D. High-grade CVG for Stabilisation Control Systems and Tactical Grade Systems. In Proceedings of the Inertial Sensors and Systems Symposium (ISS), Karlsruhe, Germany, 9 September 2013.

15. Cho, J.Y.; Woo, J.-K.; He, G.; Yang, D.; Boyd, C.; Singh, S.; Darvishian, A.; Shiari, B.; Najafi, K. 1.5-Million Q-Factor Vacuum-Packaged Birdbath Resonator Gyroscope (BRG). In Proceedings of the 2019 IEEE 32nd International Conference on Micro Electro Mechanical Systems (MEMS); Institute of Electrical and Electronics Engineers (IEEE), Seoul, Korea, 27-31 January 2019; pp. 210-213.

16. Wu, Y.; Pan, Y.; Wang, D.; Qu, T.; Huang, Y. The Study on Temperature Characteristics of a Monolithic Fused Silica Cylindrical Resonator. In Proceedings of the 2016 Joint International Information Technology, Mechanical and Electronic Engineering Conference, Xi'an, China, 4-5 October 2016.

17. Pan, Y.; Wang, D.; Wang, Y.; Liu, J.; Wu, S.; Qu, T.; Yang, K.; Luo, H. Monolithic Cylindrical Fused Silica Resonators with High Q Factors. Sensors 2016, 16, 1185. [CrossRef]

18. Luo, Y.; Qu, T.; Cui, Y.; Pan, Y.; Yu, M.; Luo, H.; Jia, Y.; Tan, Z.; Liu, J.; Zhang, B. Cylindrical Fused Silica Resonators Driven by PZT Thin Film Electrodes with Q Factor Achieving 2.89 Million after Coating. Sci. Rep. 2019, 9, 9461. [CrossRef]

19. Wang, X.; Wu, W.; Fang, Z.; Luo, B.; Li, Y.; Jiang, Q. Temperature Drift Compensation for Hemispherical Resonator Gyro Based on Natural Frequency. Sensors 2012, 12, 6434-6446. [CrossRef]

20. Wu, Y.; Xi, X.; Tao, Y.; Wu, X.; Wu, X. A Study of the Temperature Characteristics of Vibration Mode Axes for Vibratory Cylinder Gyroscopes. Sensors 2011, 11, 7665-7677. [CrossRef] 
21. Green, E.I. The story of Q. Am. Sci. 1955, 43, 584-594.

22. Li, B.; Ma, G.; Wang, C. Hemispherical Resonator Gyroscope Accuracy Analysis Under Temperature Influence. Sens. Transducers 2014, 173, 284.

23. Tang, Q.; Wang, X.; Yang, Q.; Liu, C. Static temperature analysis and compensation of MEMS gyroscopes. Int. J. Metrol. Qual. Eng. 2013, 4, 209-214. [CrossRef]

24. Shao, P. Microscale Hemispherical Shell Resonating Gyroscopes. Ph.D. Thesis, Georgia Institute of Technology, Atlanta, Georgia, 2014.

25. Zega, V.; Frangi, A.; Guercilena, A.; Gattere, G. Analysis of Frequency Stability and Thermoelastic Effects for Slotted Tuning Fork MEMS Resonators. Sensors 2018, 18, 2157. [CrossRef] [PubMed]

26. Qiu, Z.; Qu, T.; Pan, Y.; Jia, Y.; Fan, Z.; Yang, K.; Yuan, J.; Luo, H. Optical and Electrical Method Characterizing the Dynamic Behavior of the Fused Silica Cylindrical Resonator. Sensors 2019, 19, 2928. [CrossRef] [PubMed]

27. Xiao, P.; Qiu, Z.; Pan, Y.; Li, S.; Qu, T.; Tan, Z.; Liu, J.; Yang, K.; Zhao, W.; Luo, H.; et al. Influence of Electrostatic Forces on the Vibrational Characteristics of Resonators for Coriolis Vibratory Gyroscopes. Sensors 2020, 20, 295. [CrossRef]

28. Wang, Y.; Pan, Y.; Qu, T.; Jia, Y.; Yang, K.; Luo, H. Decreasing Frequency Splits of Hemispherical Resonators by Chemical Etching. Sensors 2018, 18, 3772. [CrossRef]

29. Tao, Y.; Pan, Y.; Jin, S.; Jia, Y.; Yang, K.; Luo, H. Trimming of Imperfect Cylindrical Fused Silica Resonators by Chemical Etching. Sensors 2019, 19, 3596. [CrossRef]

30. Zhang, M.; Llaser, N.; Rodes, F. High-Precision Time-Domain Measurement of Quality Factor. IEEE Trans. Instrum. Meas. 2011, 61, 842-844. [CrossRef]

31. Warburton, G.B. Vibration of Thin Cylindrical Shells. J. Mech. Eng. Sci. 1965, 7, 399-407. [CrossRef]

32. Loveday, P.; Rogers, C. Free vibration of elastically supported thin cylinders including gyroscopic effects. J. Sound Vib. 1998, 217, 547-562. [CrossRef]

33. Xi, X.; Wu, X.; Zhang, Y.; Zhou, X.; Wu, X.; Wu, Y. A study on $\mathrm{Q}$ factor of the trimmed resonator for vibratory cupped gyroscopes. Sens. Actuators A Phys. 2014, 218, 23-32. [CrossRef]

34. Tan, Y.Y.; Hong, Y.U.; Huang, Q.A.; Liu, T.Q. Effect of Temperature on the Young's Modulus of Silicon Nano-Films. Chin. J. Electron Devices 2007, 30, 755-758.

35. Li, X.; Ono, T.; Wang, Y.; Esashi, M. Study on ultra-thin NEMS cantilevers-High yield fabrication and size-effect on Young's modulus of silicon. In Proceedings of the Technical Digest. MEMS 2002 IEEE International Conference. Fifteenth IEEE International Conference on Micro Electro Mechanical Systems (Cat. No.02CH37266), Las Vegas, NV, USA, 24-24 January 2002; pp. 427-430.

36. Darvishian, A. Design and Analysis of Extremely Low-Noise MEMS Gyroscopes for Navigation. Ph.D. Thesis, University of Michigan, Ann Arbor, MI, USA, 2018.

37. National Institute of Standards and Technology (NIST) Property Data Summaries. Available online: https://srdata.nist.gov/ (accessed on 13 February 2020).

38. McSkimin, H.J. Measurement of Elastic Constants at Low Temperatures by Means of Ultrasonic Waves-Data for Silicon and Germanium Single Crystals, and for Fused Silica. J. Appl. Phys. 1953, 24, 988-997. [CrossRef]

39. Uchiyama, T.; Tomaru, T.; Tobar, M.; Tatsumi, D.; Miyoki, S.; Ohashi, M.; Kuroda, K.; Suzuki, T.; Sato, N.; Haruyama, T.; et al. Mechanical quality factor of a cryogenic sapphire test mass for gravitational wave detectors. Phys. Lett. A 1999, 261, 5-11. [CrossRef]

40. Zener, C. Elasticity and Anelasticity of Metals; University of Chicago Press: Chicago, IL, USA, 1948.

41. Lu, P.; Lee, H.; Lu, C.; Chen, H. Thermoelastic damping in cylindrical shells with application to tubular oscillator structures. Int. J. Mech. Sci. 2008, 50, 501-512. [CrossRef]

42. Wong, S.; Fox, C.; McWilliam, S. Thermoelastic damping of the in-plane vibration of thin silicon rings. J. Sound Vib. 2006, 293, 266-285. [CrossRef]

43. Sun, Y.; Tohmyoh, H. Thermoelastic damping of the axisymmetric vibration of circular plate resonators. J. Sound Vib. 2009, 319, 392-405. [CrossRef]

44. Flügge, W. Statik Und Dynamik Der Schalen; Springer: Berlin/Heidelberg, Germany, 2013.

(C) 2020 by the authors. Licensee MDPI, Basel, Switzerland. This article is an open access article distributed under the terms and conditions of the Creative Commons Attribution (CC BY) license (http://creativecommons.org/licenses/by/4.0/). 